\title{
Eosinophil chemotactic activity in bronchoalveolar lavage from idiopathic pulmonary fibrosis is dependent on cytokine priming of eosinophils
}

\author{
K.A. Boomars*, R.C. Schweizer*, P. Zanen*, J.M.M. van den Bosch**, \\ J-W.J. Lammers*, L. Koenderman*
}

Eosinophil chemotactic activity in bronchoalveolar lavage from idiopathic pulmonary fibrosis is dependent on cytokine priming of eosinophils. K.A. Boomars, R.C. Schweizer, P. Zanen, J.M.M. van den Bosch, J-W.J. Lammers, L. Koenderman. CERS Journals Ltd 1998. ABSTRACT: Increased numbers of eosinophils have been found in bronchoalveolar lavage (BAL) fluid obtained from patients with idiopathic pulmonary fibrosis (IPF). This suggests the presence of one or more cytokines in the lung tissue of patients with IPF, which are involved in the induction of migration of eosinophils towards the pulmonary compartment. To evaluate this hypothesis, we studied migratory responses of blood eosinophils towards BAL fluid.

Migratory responses were tested by means of a modified Boyden chamber assay in 21 patients with IPF and 14 healthy controls. Experiments were performed with unprimed eosinophils and in vitro primed eosinophils (preincubated with $10^{-11} \mathrm{M}$ granulocyte macrophage-colony-stimulating factor). Changes in intracellular free $\mathrm{Ca}^{2+}\left(\left[\mathrm{Ca}^{2+}\right]_{\mathrm{j}}\right)$ in eosinophils in response to $\mathrm{BAL}$ fluid were also investigated, to characterize putative chemotaxins further.

Chemotactic responses of eosinophils were observed towards BAL fluid from both patients with IPF and controls, provided that the eosinophils were primed. No changes in $\left[\mathrm{Ca}^{2+}\right]_{\mathrm{i}}$ in eosinophils were detected in response to BAL fluid. Furthermore, neither a blocking antibody against interleukin-8 nor one against regulated on activation, normal T-cell, expressed and secreted (RANTES) influenced the response.

Since a chemotactic response of in vitro primed eosinophils was also observed towards bronchoalveolar lavage fluid from normals, it was concluded, that in idiopathic pulmonary fibrosis, apart from the presence of a chemotactic factor in the lung tissue, other mechanisms such as priming of eosinophils in the peripheral blood are responsible for the extravasation of eosinophils into the pulmonary compartment. As no changes in $\left[\mathrm{Ca}^{2+}\right]$, were observed in the eosinophils after incubation with bronchoalveolar lavage fluid, the chemotaxin responsible for the migratory responses is probably not one of the known eosinophil-activating chemokines. Eur Respir J 1998; 11: 1009-1014.
*Dept of Pulmonary Diseases, University Hospital Utrecht, Utrecht, The Netherlands. **Dept of Pulmonary Diseases, St Antonius Hospital, Nieuwegein, The Netherlands.

Correspondence: L. Koenderman

Dept of Pulmonary Diseases

Room F. 02.333

University Hospital Utrecht

P.O. Box 85500

3508 GA Utrecht

The Netherlands

Fax: 0302542155

Keywords: Bronchoalveolar lavage

chemotaxis

eosinophils

idiopathic pulmonary fibrosis

Received: April 301997

Accepted after revision December 171997

This study was supported by a research grant from Glaxo Wellcome BV, The Netherlands.
An enhanced number of eosinophils is often found in the interstitium and alveolar spaces in lung biopsy specimens obtained from patients with idiopathic pulmonary fibrosis (IPF) [1, 2]. The influx of eosinophils into the pulmonary compartment has also been demonstrated in the bronchoalveolar lavage (BAL) fluid from patients with IPF [3, 4]. Several studies suggest that eosinophils may play a role in pulmonary fibrosis. A higher number of eosinophils in BAL fluid has been related to a poor response to therapy and shorter survival $[4,5]$.

In vitro studies have demonstrated that migration of eosinophils is induced by various chemokines $[6,7]$. Eosinophils isolated from the peripheral blood of healthy volunteers require in vitro priming with cytokines such as granulocyte macrophage-colony-stimulating factor (GMCSF) and interleukin (IL)-5, for optimal migratory responses toward chemokines such as IL-8 and regulated on activation, normal T-cell, expressed and secreted (RANTES)

For editorial comments see page 999.
$[7,8]$. This is in contrast to eosinophils isolated from the peripheral blood of patients with allergic asthma, which show increased spontaneous migratory responses in vitro towards various chemoattractants, indicating an in vivo primed phenotype $[9,10]$. It was demonstrated recently that eosinophils isolated from patients with asthma had a chemotactic response towards BAL fluid obtained from three patients with allergic asthma [11]. However, this study was not controlled with BAL fluid obtained from healthy controls.

In the present study, the presence of a putative chemoattractant in the lung tissue of patients with IPF was investigated using BAL fluid from this study group. The migration of eosinophils isolated from the blood of healthy volunteers towards BAL fluid obtained from patients with IPF was studied. In addition, responses were compared to those of eosinophils towards BAL fluid from healthy volunteers. In order to examine the effect of priming on the migratory responses, experiments were also carried out with eosinophils preincubated with GM-CSF. 


\section{Materials and methods}

\section{Patients and controls}

BAL fluid was obtained from 21 patients with IPF who underwent a BAL in the authors' hospital between 1987 and 1994. The diagnosis of IPF was based on compatible clinical history, evidence of diffuse interstitial infiltrates on chest radiographs and, in most patients, restrictive lung function tests. Patients with significant environmental or occupational exposure, a history of ingestion of fibrogenic drugs, extrinsic allergic alveolitis, atopy, left ventricular failure or collagen vascular diseases were excluded. Lung tissue for histological confirmation of the diagnosis of IPF was obtained by open lung biopsy. Patients did not receive corticosteroid or immunosuppressive therapy before or at the time of lavage. The control group consisted of 14 healthy volunteers without chest abnormalities or pulmonary disease. The characteristics of patients and controls are listed in table 1 . This study was approved by the hospital's medical Ethics Committee.

\section{Bronchoalveolar lavage}

Bronchoalveolar lavage was performed as described previously during fibreoptic bronchoscopy [4]. Blood samples were taken at the same time. In brief, the lavage procedure was as follows. BAL was performed by standardized washing of the middle lobe with four $50 \mathrm{~mL}$ aliquots of sterile saline solution $(0.9 \% \mathrm{NaCl})$ at room temperature. Lavage fluid samples, kept on ice in a siliconized specimen trap, were centrifuged $(10 \mathrm{~min}, 350 \times \mathrm{g})$ immediately after the lavage procedure, and separated into cells and supernatant. The cells from the last three aliquots were pooled, washed twice, counted and suspended in minimal essential medium (Gibco, Grand Island, NY, USA) supplemented with $1 \%$ bovine serum albumin (Organon, Teknika, Boxtel, The Netherlands). Preparations of the cell suspensions were made in a cytocentrifuge (Shandon, Runcorn, UK). Cytospin slides of BAL cells were stained with MayGrünwald-Giemsa (Merck, Darmstadt, Germany) for differential cell counts. At least 1,000 cells were counted. Supernatants were stored frozen at $-70^{\circ} \mathrm{C}$.

\section{Reagents}

Platelet-activating factor (PAF; 1-O-hexadecyl-2-acetyl-sn-glycero-3-phosphorylcholine) was purchased from Sigma (St Louis, MO, USA). Ficoll-paque and Percoll were obtained from Pharmacia (Uppsala, Sweden). All other materials were reagent grade. Experiments were performed in incubation buffer containing: $132 \mathrm{mmol} \cdot \mathrm{L}^{-1}$ $\mathrm{NaCl}, 6.0 \mathrm{mmol} \cdot \mathrm{L}^{-1} \mathrm{KCl}, 1.0 \mathrm{mmol} \cdot \mathrm{L}^{-1} \mathrm{CaCl}, 1.0 \mathrm{mmol} \cdot \mathrm{L}^{-1}$ $\mathrm{MgSO}_{4}, 1.2 \mathrm{mmol} \cdot \mathrm{L}^{-1} \mathrm{KH}_{2} \mathrm{PO}_{4}, 20 \mathrm{mmol} \cdot \mathrm{L}^{-1} \mathrm{~N}$-2-hydroxyethylpiperazine-N-2-ethanesulphonic acid (HEPES), 5 glucose $\mathrm{mmol} \cdot \mathrm{L}^{-1}$ and $1.0 \%$ human serum albumin (HSA) weight/volume (w/v).

\section{Cytokines and antibodies}

Recombinant human (rH) GM-CSF and rH IL-8 were obtained from Genzyme (Cambridge, MA, USA). Rabbit (polyclonal) anti-human IL-8 antibody was purchased from Biosources International (Camarillo, USA). Mouse (monoclonal) anti-human RANTES antibody was obtained from R\&D Systems (Minneapolis, USA). Both blocking antibodies were titrated and blocked at a concentration of $\breve{S} 10 \mu \mathrm{g} \cdot \mathrm{mL}^{-1}$ and $\breve{S} 50 \mu \mathrm{g} \cdot \mathrm{mL}^{-1}$ for IL-8 and RANTES, respectively. Cytokines and antibodies were stored at $-80^{\circ} \mathrm{C}$. IL-8 enzyme-linked immunosorbent assay (ELISA) was obtained from Central Laboratory of The Netherlands Red Cross Blood Transfusion Service (Amsterdam, The Netherlands).

\section{Cell isolation}

Blood was obtained from healthy volunteers from the Blood Bank (Utrecht, The Netherlands). Eosinophils were isolated as described previously [7]. In brief, mixed granulocytes were isolated from the buffy-coat of $500 \mathrm{~mL}$ of blood anticoagulated with $0.4 \%(\mathrm{w} / \mathrm{v})$ trisodium citrate ( $\mathrm{pH}$ 7.4) as described previously [12]. To isolate eosinophils from this mixed granulocyte population, the cells were subjected to discontinuous Percoll gradient (1.084$\left.1.1 \mathrm{~g} \cdot \mathrm{mL}^{-1}\right)$ centrifugation. Eosinophils were subsequently isolated by the method described by Hansel et al. [13], removing neutrophils coated with CLB-FcR-gran 1 with immunomagnetic dynabeads [14]. The purity of the eosinophils was always $>95 \%$ and the viability was $>98 \%$.

\section{Migration assay}

Eosinophil migration was measured by a modification of the method according to BOYDEN [15] using a 48-well microchemotaxis chamber (Neuroprobe, Cabin John, MD, USA). Chemotaxins, BAL fluid or incubation buffer (30 $\mu \mathrm{L})$ were added to the lower compartments. Two filters were placed between lower and upper compartments. The lower filter had a pore width of $0.45 \mu \mathrm{m}$ (Millepore, Bedford, MA, USA, type HA) and the upper filter (cellulose nitrate) a pore width of $8 \mu \mathrm{m}$ (thickness $150 \mu \mathrm{m}$; Sartorius, Göttingen, Germany; type SM 113). The filters were soaked in incubation buffer before use. Purified eosinophils were placed in the upper compartments $(25 \mu \mathrm{L} 5 \times$ $10^{6}$ cells $\left.\cdot \mathrm{mL}^{-1}\right)$. In the priming experiments eosinophils were preincubated for $30 \mathrm{~min}$ at $37^{\circ} \mathrm{C}$ with $10^{-11} \mathrm{M} \mathrm{GM}$ CSF before the chemotaxis assay was carried out. The chambers were subsequently incubated for $2.5 \mathrm{~h}$ at $37^{\circ} \mathrm{C}$. The upper filters were removed, fixed in butanol/ethanol $(20 / 80, \mathrm{v} / \mathrm{v})$ for $10 \mathrm{~min}$ and stained with Weigert solution

Table 1. - Characteristics of patients with idiopathic pulmonary fibrosis (IPF) and control subjects

\begin{tabular}{lccccccccc}
\hline Group & $\mathrm{n}$ & $\begin{array}{c}\text { Age } \\
\text { yrs }\end{array}$ & Range & Female & Male & Nsm & Sm & $\begin{array}{c}\text { Eosinophil } \\
\text { BAL \% }\end{array}$ & Range \\
\hline IPF & 21 & 60 & $45-73$ & 9 & 12 & 14 & 7 & $12 *$ & $0.5-45.3$ \\
Control & 14 & 24 & $19-30$ & 8 & 6 & 8 & 6 & $<1$ & $0.0-0.8$ \\
\hline
\end{tabular}

Nsm: nonsmokers; Sm: smokers; BAL: bronchoalveolar lavage. *: p<0.05 IPF versus Controls (Mann-Whitney U-test). 
(composition: 1\% (v/v) haematoxylin in ethanol mixed with a $70 \mathrm{mM}$ acidic $\mathrm{FeCl}_{3}$ solution at a 1:1 ratio). The filters were dehydrated with ethanol, made transparent with xylene and fixed upside down. Migration of cells used to be quantified by the determination of the number of cells per 10 high-power fields (HPF) by light microscopy (magnification $\times 400$ ). In this way the number of cells (the leading front) that passed the upper filter (and migrated 150 $\mu \mathrm{m})$ was determined.

Recently, a new method for quantification of cell migration was developed. Migratory responses of eosinophils were quantified with an image analysis system (Quantimet 570C, Leica Cambridge Ltd, Weitzlar, Germany) using Quantimet 570 Control Software (QUIC, version 2.02) and a custom-made software programme. An automated microscope, Leitz DMRXE (Leica) was used to step through the filters in the $Z$ direction with 17 intervals of $10 \mu \mathrm{m}$. Eosinophils were counted at each level and the total migration at each level was calculated. The results were expressed as migratory index $\left(\mu \mathrm{m} \cdot\right.$ cell $\left.^{-1}\right)$, which was calculated by the cumulative migration of all intervals $(\mu \mathrm{m})$ divided by the total number of cells. In this index the cells at level $0 \mu \mathrm{m}$ were not included in the calculation. The mean of four randomly chosen points on each filterspot was calculated. Measurement of eosinophil migration towards BAL fluid obtained from patients with IPF and normals was performed by the HPF method. The chequerboard analysis and the eosinophil migration assays using BAL fluid with and without a blocking polyclonal antibody against IL-8 and with and without a blocking monoclonal antibody against RANTES, were analysed by the recently developed image analysis set-up, described above.

\section{$\left[\mathrm{Ca}^{2+}\right]_{i}$ measurement}

The intracellular free $\mathrm{Ca}^{2+}$ concentration $\left(\left[\mathrm{Ca}^{2+}\right]_{\mathrm{i}}\right)$ was measured as described previously [16]. In brief, eosinophils $\left(10^{7} \cdot \mathrm{mL}^{-1}\right)$ were preincubated with $1.5 \mu \mathrm{mol} \cdot \mathrm{L}^{-1}$ Indo-1/acetoxy-methyl ester (AM) in HEPES buffer for $45 \mathrm{~min}$ at $37^{\circ} \mathrm{C}$. Subsequently, cells were washed, resuspended in incubation buffer and kept at room temperature in the dark until use. Fluorescence measurements were performed in a fluorospectrophotometer (Hitachi F-3000, Tokyo, Japan) at $37^{\circ} \mathrm{C}$ in stirred suspensions. Calibration of Indo- 1 fluorescence as a function of $\left[\mathrm{Ca}^{2+}\right]_{i}$, was performed as follows: to saturate all trapped Indo- 1 with $\mathrm{Ca}^{2+}, 5$ $\mu \mathrm{mol} \cdot \mathrm{L}^{-1}$ digitonin was added to the cell suspensions. Subsequently, the $\mathrm{Ca}^{2+}$-dependent Indo-1 signal was quenched by adding $0.5 \mathrm{mmol} \cdot \mathrm{L}^{-1} \mathrm{Mn} 2+.\left[\mathrm{Ca}^{2+}\right]_{\mathrm{i}}$ was calculated as described elsewhere [17], using $250 \mathrm{nmol} \cdot \mathrm{L}^{-1}$ as the dissociation constant $\left(\mathrm{k}_{\mathrm{d}}\right)$ for the Indo-1/Ca ${ }^{2+}$ complex [18]. After each experiment, $1 \mu \mathrm{mol} \cdot \mathrm{L}^{-1} \mathrm{PAF}$ was added to the cells as a positive control.

\section{Measurement of interleukin-8 in bronchoalveolar lavage fluid}

IL-8 levels were determined in BAL fluid obtained from patients with IPF and control subjects by means of a sandwich ELISA, according to the standard procedure provided by the manufacturer (obtained from Central Lab- oratory of The Netherlands Red Cross Blood Transfusion Service, Amsterdam, The Netherlands).

\section{Statistical analysis}

Statistical evaluation of the migratory responses was performed by repeated-measurements analysis of variance. When significant differences were found the protected least squares difference (LSD)-test was used. The MannWhitney U-test was used to evaluate the differences in the percentages of eosinophils and IL-8 levels in BAL fluid between patients with IPF and healthy controls. A p-value $<0.05$ was considered to be significant.

\section{Results}

Chemotaxis of eosinophils induced by bronchoalveolar lavage fluid requires priming

The migratory responses of eosinophils derived from normal donors towards BAL fluid obtained from patients with IPF and healthy control subjects were compared. Mig-
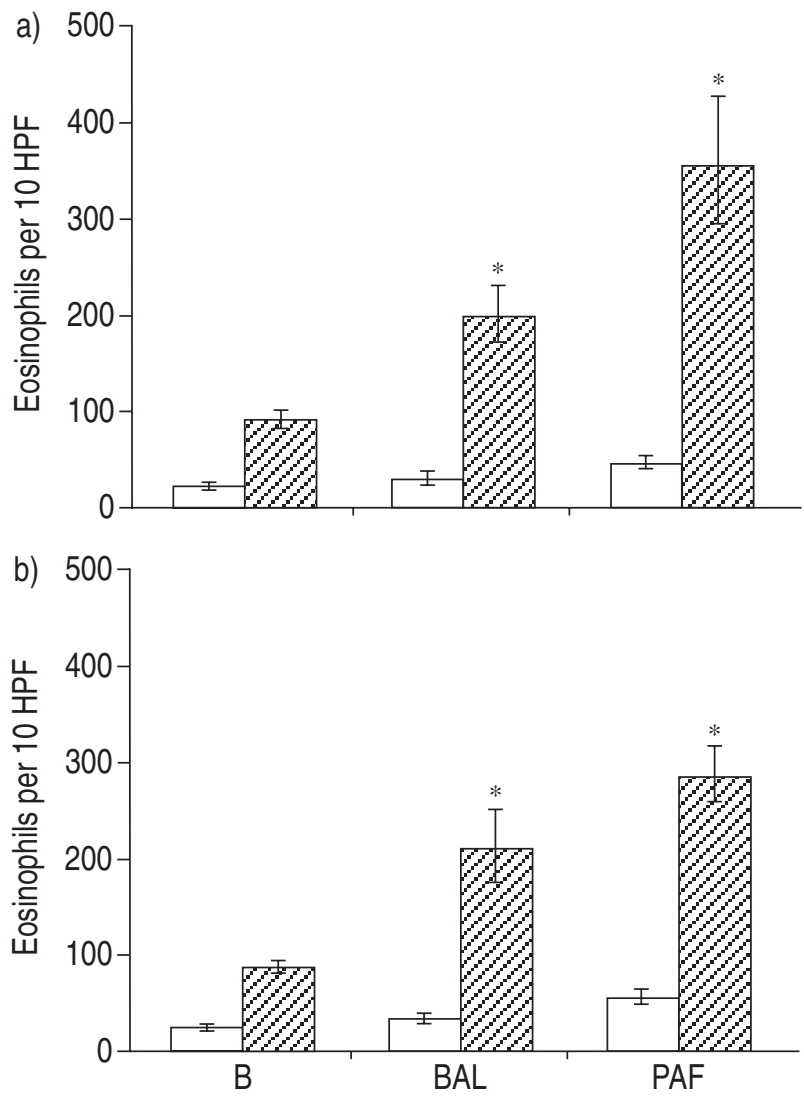

Fig. 1. - Migration of human eosinophils induced by bronchoalveolar lavage (BAL) fluid obtained from: a) patients with idiopathic pulmonary fibrosis $(n=21)$; and $b)$ healthy controls $(n=14)$. Experiments were performed with unprimed eosinophils $(\square)$ and eosinophils primed with granulocyte macrophage-colony stimulating factor $\left(10^{-11} \mathrm{~mol} \cdot \mathrm{L}^{-1}, 30\right.$ $\left.\min , 37^{\circ} \mathrm{C}\right)(\angle 2)$. Platelet-activating factor $\left(\mathrm{PAF}, 10^{-8} \mathrm{~mol} \cdot \mathrm{L}^{-1}\right)$ was used as a positive control. B: buffer control; HPF: high-power field. Data are expressed as mean values \pm SEM. *: $\mathrm{p}<0.05$, significant difference from buffer control. 
ratory responses from eosinophils towards BAL fluid obtained from patients with IPF are demonstrated in fig. 1a, while responses of eosinophils towards BAL fluid obtained from control subjects are shown in fig. 1b. Unprimed eosinophils did not show a chemotactic response towards BAL fluid from either patients with IPF or healthy controls. Interestingly, eosinophils that were preincubated with $10^{-11}$ M GM-CSF (for $30 \mathrm{~min}$ at $37^{\circ} \mathrm{C}$ ) responded significantly towards BAL fluid obtained from patients with IPF when compared with HEPES buffer. Comparable chemotactic responses of cytokine-primed eosinophils were observed towards BAL fluid obtained from healthy controls. A chequerboard analysis demonstrated that migration of primed eosinophils induced by BAL fluid was caused by chemotaxis rather than by chemokinetic activation (table 2). This was found for BAL fluid obtained from both patients with IPF and control subjects.

Changes in $\left[\mathrm{Ca}^{2+}\right]_{i}$ in eosinophils induced by bronchoalveolar lavage fluid

Figure 2 shows the effects of BAL on $\left[\mathrm{Ca}^{2+}\right]_{\mathrm{i}}$ measurements in eosinophils in response to BAL fluid obtained from patients with IPF and healthy controls. BAL fluid was diluted 10× with HEPES buffer before it was added to the cells (1:100 final concentration), at the indicated timepoints. In these experiments $1 \mu \mathrm{mol} \cdot \mathrm{L}^{-1} \mathrm{PAF}$ was used as a positive control. BAL fluid obtained from patients with IPF was not able to induce a rise in $\left[\mathrm{Ca}^{2+}\right]_{\mathrm{i}}$ in eosinophils. Priming of the eosinophils with 10-11 M GM-CSF did not change these results. No differences were observed between the data obtained in response to BAL fluid from patients with IPF and BAL fluid obtained from healthy controls. Furthermore, responses were similar when undiluted BAL fluid was added to the assay (results not shown).

\section{Determination of interleukin-8 levels in bronchoalveolar lavage fluid}

To determine whether this chemokine may be responsible for chemotaxis of eosinophils, IL-8 levels were measured in BAL fluid from patients with IPF and healthy

Table 2. - Chequerboard analysis of migration of primed eosinophils, induced by bronchoalveolar lavage fluid obtained from a patient with idiopathic pulmonary fibrosis

\begin{tabular}{lccccc}
\hline & & \multicolumn{4}{c}{ Lower compartment } \\
\cline { 3 - 6 } & & Buffer & BALF & BALF & BALF \\
& Dilution & & $1: 4$ & $1: 2$ & undiluted \\
\hline Upper compartment & & 22.8 & 24.8 & 50.0 & 47.8 \\
Buffer & $1: 4$ & 31.6 & 31.3 & 30.5 & 34.7 \\
BALF & $1: 2$ & 18.8 & 17.9 & 23.3 & 26.3 \\
BALF &
\end{tabular}

Bronchoalveolar lavage fluid (BALF) was placed in the lower compartment of the Boyden chamber (normal migration assay), in the upper compartment or in both compartments in a dilution range. The data are presented as migratory index $\left(\mu \mathrm{m} \cdot \mathrm{cell}^{-1}\right)$ and are representative for two additional experiments. Priming of eosinophils was carried out by preincubation of the cells with granulocyte macrophage-colony stimulating factor $10^{-11} \mathrm{~mol} \cdot \mathrm{L}^{-1}$, $30 \min , 37^{\circ} \mathrm{C}$

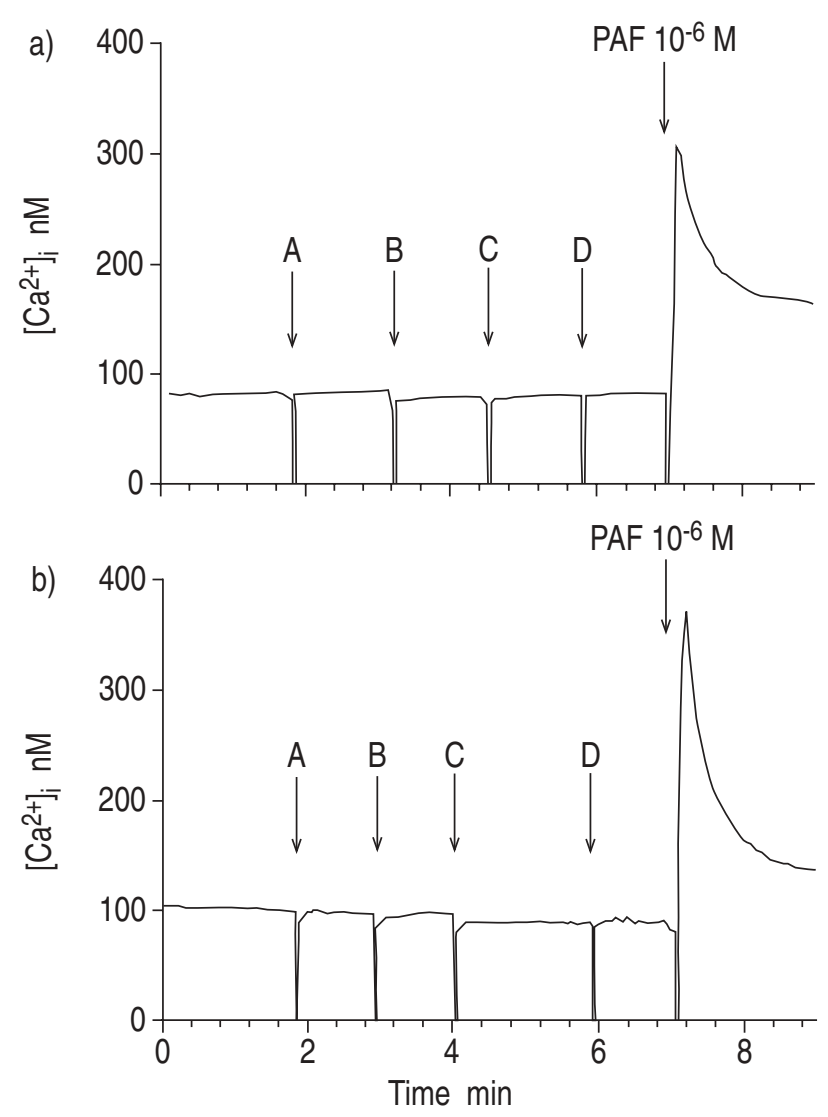

Fig. 2. - Responses of bronchoalveolar lavage (BAL) fluid obtained from patients with idiopathic pulmonary fibrosis (IPF) and healthy controls, on $\left[\mathrm{Ca}^{2+}\right]_{\mathrm{i}}$ in human eosinophils loaded with Indo-1/AM. Experiments were performed with a) unprimed eosinophils and b) eosinophils primed with granulocyte macrophage-colony stimulating factor $\left(10^{-11} \mathrm{~mol} \cdot \mathrm{L}^{-1}\right.$, for $30 \mathrm{~min}$ at $\left.37^{\circ} \mathrm{C}\right)$. BAL fluid $(100 \times$ dilution final concentration) was added to the cells at the indicated time-points. After each experiment, $1 \mu \mathrm{mol} \cdot \mathrm{L}^{-1}$ PAF was used as a positive control. A: IPF patient 1 ; B: IPF patient 2; C: Control 1; D: Control 2. The results shown are representative for two other experiments, in which BAL fluid obtained from other patients and controls showed similar results.

Table 3. - Interleukin-8 levels in bronchoalveolar lavage fluid obtained from patients with idiopathic pulmonary fibrosis (IPF) and healthy controls

\begin{tabular}{lccc}
\hline & \multicolumn{3}{c}{ IL-8 $\mathrm{pg} \cdot \mathrm{mL}^{-1}$} \\
\cline { 2 - 4 } & $\mathrm{n}$ & Mean & SEM \\
\hline IPF & 17 & $98.5^{*}$ & 36.8 \\
Controls & 12 & 10.8 & 1.4
\end{tabular}

IL-8: interleukin-8; n: number of cases; *: $\mathrm{p}<0.05$ (Mann-Whitney U-test, IPF versus controls).

controls. IL-8 levels in BAL fluid obtained from patients with IPF were significantly increased compared with those taken from controls $(\mathrm{p}<0.05$; table 3$)$.

Effect of blocking antibodies on bronchoalveolar lavage fluid-induced eosinophil chemotaxis

Since IL- 8 does not evoke a change in $\left[\mathrm{Ca}^{2+}\right]_{\mathrm{i}}$ in primed eosinophils and shares these characteristics with the chemoattractive factor found in BAL fluid, the migration of unprimed and primed eosinophils towards BAL fluid was studied in the presence of a blocking polyclonal antibody 
Table 4. - Migratory responses of primed eosinophils induced by bronchoalveolar lavage fluid (BALF) obtained from patients with idiopathic pulmonary fibrosis, in the absence or presence of blocking anti-interleukin (IL)-8 or anti-regulated on activation, normal T-cell, expressed and secreted (RANTES) antibodies

\begin{tabular}{lcccc}
\hline & Buffer & BALF & $\begin{array}{c}\text { BALF+ } \\
\text { anti-IL-8 }\end{array}$ & $\begin{array}{c}\text { BALF+ } \\
\text { anti- } \\
\text { RANTES }\end{array}$ \\
\hline MI & $23.2 \pm 1.7$ & $41.3 \pm 3.0$ & $43.1 \pm 2.9$ & \\
MI & $28.3 \pm 0.6$ & $37.8 \pm 2.1$ & & $42.8 \pm 4.0$ \\
\hline
\end{tabular}

BALF was treated in the presence or absence of anti-IL-8 (10 $\left.\mu \mathrm{g} \cdot \mathrm{mL}^{-1}\right)$ or anti-RANTES $\left(50 \mu \mathrm{g} \cdot \mathrm{mL}^{-1}\right)$ for $15 \mathrm{~min}$ at room temperature. Hereafter, chemotaxis was measured with eosinophils primed with granulocyte macrophage colony-stimulating factor (see legend for table 2). Data are expressed as mean \pm SEM $(\mathrm{n}=5)$ and migratory index (MI) is expressed in $\mu \mathrm{m} \cdot \mathrm{cell}^{-1}$.

directed against IL-8 $\left(10 \mu \mathrm{g} \cdot \mathrm{mL}^{-1}\right)$. The blocking antibody against IL-8 was tested in a migration assay with eosinophils and found to be potent in blocking migration towards IL-8 (results not shown). The chemotaxis of primed eosinophils towards BAL fluid from patients with IPF was not blocked when anti-IL-8 was added to the BAL fluid (table 4). Data obtained in experiments with BAL fluid from healthy controls showed similar results.

To analyse the possible role of RANTES, the migration of unprimed and primed eosinophils towards BAL fluid was studied in the presence of a blocking monoclonal antibody directed against RANTES $\left(50 \mu \mathrm{g} \cdot \mathrm{mL}^{-1}\right)$. The blocking antibody against RANTES was tested in a migration assay with eosinophils and found to be potent in blocking migration towards RANTES (results not shown). The chemotaxis of primed eosinophils induced by BAL fluid from patients with IPF was not blocked when anti-RANTES was added to the BAL fluid (table 4). Similar results were obtained in experiments with BAL fluid from healthy controls (data not shown).

\section{Discussion}

In this study an enhanced number of eosinophils was demonstrated in the BAL fluid obtained from patients with IPF, in agreement with previous reports [3, 4, 19, 20]. Cytokines present in the lung tissue of patients with IPF may be responsible for the induction of chemotaxis of eosinophils to the pulmonary compartment. To evaluate this hypothesis, migratory responses of eosinophils induced by BAL fluid of patients with IPF and healthy controls were studied.

Significant migratory responses of eosinophils towards BAL fluid from patients with IPF and BAL fluid from healthy controls were observed, provided that the eosinophils were primed with cytokines. It has been demonstrated that eosinophils derived from peripheral blood from normals require in vitro priming with cytokines such as GM-CSF or IL-5 for optimal migratory responses towards PAF, formyl-methionyl-leucyl-phenylalanine (FMLP) and the chemokines RANTES and IL-8 [6, 7, 21]. Cell migration can be divided into chemokinesis (random movement) and chemotaxis (locomotory response of cells to concentration gradients of chemotactic molecules). The differentiation between chemotaxis and chemokinesis was investigated using a chequerboard analysis. These results demonstrated the presence of a chemoattractant rather than a chemokinetic mediator. Therefore, cytokines such as IL-5 in BAL fluid are unlikely candidates, since IL-5 induces mainly chemokinesis [22].

To characterize further the identity of the chemoattractant changes in the $\left[\mathrm{Ca}^{2+}\right]_{\mathrm{i}}$ in eosinophils in response to BAL fluid from patients with IPF and controls were investigated. However, no changes in the $\left[\mathrm{Ca}^{2+}\right]_{\mathrm{i}}$ in response to BAL fluid were observed. This seems to rule out several potent eosinophil chemotaxins such as PAF, complement fragment 5a (C5a) and pro-inflammatory members of the chemokine family (including eotaxin) that all induce increases in $\left[\mathrm{Ca}^{2+}\right]_{i}$ in eosinophils $[7,23]$. Recently, expression of RANTES protein and RANTES messenger ribonucleic acid has been demonstrated in BAL cells obtained from patients with IPF and controls [24]. However, no correlation was found between the number of eosinophils in BAL fluid and the expression of RANTES in BAL cells. In the present study, migratory activity of primed eosinophils towards BAL fluid from patients with IPF was not blocked when a blocking antibody directed against RANTES was added to the BAL fluid.

It was considered that IL- 8 may be responsible for the migratory responses of eosinophils towards BAL fluid from patients with IPF, since priming of eosinophils is required for chemotaxis of eosinophils towards IL-8, and IL-8 is not able to induce an appreciable rise in $\left[\mathrm{Ca}^{2+}\right]_{i}$, in either unprimed or primed eosinophils [7]. However, the migratory response of eosinophils towards BAL fluid from patients with IPF could not be blocked with a blocking antibody directed against IL- 8 . The concentration of IL-8 measured in BAL fluid from patients with IPF was found to be increased compared with normals, as was previously described by two other groups $[25,26]$. Although the concentration of IL-8 measured in BAL fluid of patients with IPF and normals differs among the three studies, the concentration found in all studies seems to be too low to induce chemotaxis of eosinophils in vitro. However, the possibility of synergism of several chemotactic factors in a very low concentration in BAL fluid from patients with IPF and controls cannot be excluded by the results of this study.

In this study, no significant difference was found between the chemotactic response of in vitro primed eosinophils towards BAL fluid obtained from patients with IPF and BAL fluid from normals. This implies that besides chemotaxis, other mechanisms must play a role in the extravasation of eosinophils. Since migration of eosinophils towards BAL fluid required priming with GM-CSF or IL-5, priming in vivo is critical for eosinophil responsiveness towards the chemotaxins present in BAL. Interestingly, it has already been demonstrated that eosinophils isolated from the peripheral blood of asthmatic patients and patients with atopic dermatitis do not require in vitro priming for a migratory response towards several cytokines or BAL fluid from patients with asthma [9, 11, 21]. Under these circumstances priming has occurred in the peripheral blood in vivo. In addition to chemotaxis, adhesion of the eosinophils to the vessel wall involves the expression of relevant adhesion molecules on the endothelial cells and the eosinophils. In allergic inflammation upregulation of various adhesion molecules has been observed in bronchial biopsies [27]. 
This study demonstrated chemotactic responses of in vitro primed eosinophils towards bronchoalveolar lavage fluid, not only from idiopathic pulmonary fibrosis but also from normals, indicating the presence of a chemoattractant in the pulmonary compartment of healthy individuals. This chemotaxin might be involved in normal immune surveillance in the pulmonary compartment. In bronchoalveolar lavage fluid from healthy subjects, however, hardly any eosinophils are found. It is tempting to speculate that during the disease process in idiopathic pulmonary fibrosis, the chemoattractant causes extravasation of eosinophils into the lung, provided that eosinophils are primed in the peripheral blood and the relevant adhesion molecules are expressed on both eosinophils and endothelial cells. Further studies, for example a migration assay using eosinophils from the peripheral blood from patients with idiopathic pulmonary fibrosis, are needed to validate this hypothesis.

\section{References}

1. Dunnill MS. Pulmonary fibrosis. Histopathology 1990; 16: 321-329.

2. Katzenstein AL. Pathogenesis of "fibrosis" in interstitial pneumonia: an electron microscopic study. Hum Pathol 1985; 16: 1015-1024.

3. BAL Cooperative Group Steering Committee. Bronchoalveolar lavage constituents in healthy individuals, idiopathic pulmonary fibrosis, and selected comparison groups. Am Rev Respir Dis 1990; 141: S169-S202.

4. Boomars KA, Wagenaar SjSc, Mulder PHG, van VelzenBlad H, van den Bosch JMM. Relationship between cells obtained by bronchoalveolar lavage fluid and survival in idiopathic pulmonary fibrosis. Thorax 1995; 50: 10871092.

5. Rudd RM, Haslam PL, Turner-Warwick M. Cryptogenic fibrosing alveolitis: relationships of pulmonary physiology and bronchoalveolar lavage to response to treatment and prognosis. Am Rev Respir Dis 1981; 124: 1-8.

6. Warringa RA, Koenderman L, Kok PT, Kreukniet J, Bruijnzeel PL. Modulation and induction of eosinophil chemotaxis by granulocyte-macrophage colony-stimulating factor and interleukin-3. Blood 1991; 77: 2694-2700.

7. Schweizer RC, Welmers BA, Raaijmakers JA, Zanen P, Lammers JW, Koenderman L. RANTES- and interleukin-8-induced responses in normal human eosinophils: effects of priming with interleukin-5. Blood 1994; 83: 3697-3704.

8. Murphy PM, Tiffany HL. Cloning of complementary DNA encoding a functional human interleukin-8 receptor. Science 1991; 253: 1280-1283.

9. Warringa RA, Mengelers HJ, Rauijmakers JA, Bruijnzeel PL, Koenderman L. Upregulation of formyl-peptide and interleukin-8-induced eosinophil chemotaxis in patients with allergic asthma. J Allergy Clin Immunol 1993; 91: 1198-1205.

10. Shemi R, Cromwell O, Wardlaw A, Moqbel R, Kay A. Interleukin- 8 is a chemoattractant for eosinophils purified from subjects with blood eosinophilia but not from normal healthy subjects. Clin Exp Allergy 1993; 23: 10271036.

11. Alam R, York J, Boyars M, et al. Increased MCP-1,
RANTES, and MIP-1a in bronchoalveolar lavage fluid of allergic asthmatic patients. Am J Respir Crit Care Med 1996; 153: 1398-1404.

12. Koenderman L, Kok PT, Hamelink ML, Verhoeven AJ, Bruijnzeel PL. An improved method for the isolation of eosinophilic granulocytes from peripheral blood of normal individuals. J Leukoc Biol 1988; 44: 79-86.

13. Hansel TT, Pound JD, Pilling D, et al. Purification of human blood eosinophils by negative selection using immunomagnetic beads. J Immunol Methods 1989; 122: 97-103.

14. Hartnell A, Kay AB, Wardlaw AJ. IFN-gamma induces expression of Fc gamma RIII (CD16) on human eosinophils. J Immunol 1992; 148: 1471-1478.

15. Boyden S. The chemotactic effect of mixtures of antibody and antigen on polymorphonuclear leukocytes. $J$ Exp Med 1962; 115: 453-466.

16. Koenderman L, Tool AT, Roos D, Verhoeven AJ. Priming of the respiratory burst in human eosinophils is accompanied by changes in signal transduction. J Immunol 1990; 145: 3883-3888.

17. Bijsterbosch MK, Rigley KP, Klaus GG. Cross-linking of surface immunoglobulin on B lymphocytes induces both intracellular $\mathrm{Ca}^{2+}$ release and $\mathrm{Ca}^{2+}$ influx: analysis with indo-1. Biochem Biophys Res Commun 1986; 137: 500506.

18. Grynkiewicz G, Poenie M, Tsien RY. A new generation of $\mathrm{Ca}^{2+}$ indicators with greatly improved fluorescence properties. J Biol Chem 1985; 260: 3440-3450.

19. Peterson MW, Monick M, Hunninghake GW. Prognostic role of eosinophils in pulmonary fibrosis. Chest 1987; 92 : 51-56.

20. Haslam PL, Turton CWG, Lukoszek A, et al. Bronchoalveolar lavage fluid cell counts and their relation to therapy. Thorax 1980; 35: 328-339.

21. Warringa RA, Mengelers HJ, Kuijper PH, Raaijmakers JA, Bruijnzeel PL, Koenderman L. In vivo priming of platelet-activating factor-induced eosinophil chemotaxis in allergic asthmatic individuals. Blood 1992; 79: 18361841.

22. Schweizer RC, van Kessel-Welmers BAC, Warringa RAJ, et al. Mechanisms involved in eosinophil migration. Platelet-activating factor-induced chemotaxis and interleukin-5-induced chemokinesis are mediated by different signals. J Leukoc Biol 1996; 59: 347-356.

23. Ponath P, Qin S, Ringler D, et al. Cloning of the human eosinophil chemoattractant, eotaxin. J Clin Invest 1996; 97: 604-612.

24. Petrek M, Pantelidis P, Southcott AM, et al. The source and role of RANTES in interstitial lung disease. Eur Respir J 1997; 10: 1207-1216.

25. Walker C, Bauer B, Braun RK, et al. Activated T-cells and cytokines in bronchoalveolar lavages from patients with various lung diseases associated with eosinophilia. Am J Respir Crit Care Med 1994; 150: 1038-1048.

26. Car BD, Meloni F, Luisetti M, Semenzato G, Gialdroni Grassi G, Walz A. Elevated IL-8 and MCP-1 in the bronchoalveolar lavage fluid of patients with idiopathic pulmonary fibrosis and pulmonary sarcoidosis. Am J Respir Crit Care Med 1994; 149: 655-659.

27. Gosset P, Leblond T, Janin A, et al. Expression of E-selectin, ICAM-1 and VCAM-1 on bronchial biopsies from allergic and non-allergic asthmatic patients. Int Arch Allergy Immunol 1995; 106: 69-77. 